\title{
Generation of high-power terahertz pulses in a prism
}

\author{
M. B. Johnston \\ Department of Physics, Cavendish Laboratory, University of Cambridge, Madingley Road, Cambridge CB3 OHE, UK
}

D. M. Whittaker

Department of Physics and Astronomy, University of Sheffield, Sheffield S3 7RH, UK

A. Dowd, A. G. Davies, E. H. Linfield, X. Li, and D. A. Ritchie

Department of Physics, Cavendish Laboratory, University of Cambridge, Madingley Road, Cambridge CB3 OHE, UK

Received April 16, 2002

\begin{abstract}
A compact, high-power emitter of half-cycle terahertz $(\mathrm{THz})$ radiation is demonstrated. The device consists of an epitaxial InAs emitter upon a GaAs prism and produces THz pulses that are 20 times more powerful than those from conventional planar InAs emitters. This improvement is a direct result of reorienting the transient $\mathrm{THz}$ dipole such that its axis is not perpendicular to the emitting surface. (C) 2002 Optical Society of America

OCIS codes: $230.6080,320.7080,260.3090$.
\end{abstract}

The terahertz $(\mathrm{THz})$ region of the electromagnetic spectrum $\left(\approx 0.1-\approx 10 \times 10^{12} \mathrm{~Hz}\right)$ has potential applications in many fields of science and technology, from semiconductor physics ${ }^{1,2}$ and chemical spectroscopy ${ }^{3}$ through medical imaging. ${ }^{4}$ However, exploitation of this potential has been limited by the lack of high-power coherent sources: The $\mathrm{THz}$ range falls in the gap between electron transit-time devices operating at microwave frequencies and lasers operating in the mid infrared.

A solution to this problem has been the development of pulsed generation methods in which halfcycle pulses of $\mathrm{THz}$ radiation are emitted when a semiconductor surface ${ }^{5,6}$ or heterostructure ${ }^{7}$ is illuminated with a subpicosecond near-infrared laser pulse. The $\mathrm{THz}$ pulses provide a source of coherent broadband radiation, in the range $100 \mathrm{GHz}-30 \mathrm{THz}$, which can be time resolved with femtosecond precision (in contrast to continuous-wave sources such as molecular gas and quantum cascade lasers ${ }^{8}$ ). Here we show that the $\mathrm{THz}$ power generated by this technique can be increased by more than an order of magnitude by fabrication of an emitter within a prism rather than by use of a planar geometry. This increase is comparable to or greater than that obtained by application of a magnetic field parallel to a planar surface..$^{9-14}$

$\mathrm{THz}$ emission from a semiconductor surface occurs when an above-bandgap optical pulse is incident upon a semiconductor. Most of the laser power is absorbed in a thin layer below the surface, creating a large number of electron-hole pairs. The electron and hole populations then separate, driven either by the surface electric field, ${ }^{5,15}$ as in GaAs, or by the different mobilities of the two types of carrier, as in InAs (the photo-Dember effect $^{16,17}$ ). In both cases the resultant transient electric dipole, oriented perpendicularly to the surface, undergoes half a cycle of oscillation, typically at $\mathrm{THz}$ frequencies, and thus radiates a broadband $\mathrm{THz}$ pulse.

The problem of extracting such radiation generated within a material of high refractive index $n$ is well known. For a planar surface, refraction theory shows that all rays outside an emission cone of half-angle $\alpha=\sin ^{-1}(1 / n)$ to the surface normal suffer total internal reflection and do not escape from the device. For $n=3.6$, which is appropriate for $\mathrm{GaAs}$ at $\mathrm{THz}$ frequencies, $\alpha$ is just $16^{\circ}$, which gives an emission cone covering less than $2 \%$ of the $4 \pi$ sr of solid angle potentially available. The fraction of power that is extracted depends on the orientation of the dipole axis relative to the surface: The worst case is a perpendicular dipole, as in the $\mathrm{THz}$ generation process described above. Here, as the dipole emission has the form $\sin ^{2} \theta$, where $\theta$ is the angle between the emission direction and the axis, little power is radiated into the $16^{\circ}$ emission cone. Putting these factors together, and accounting for the Fresnel reflectivity of the surface within the emission cone, means that only $0.1 \%$ of the emitted power will escape. It is therefore highly beneficial to reorient the dipole such that the emission cone will have access to a more favorable part of the $\sin ^{2} \theta$ distribution: For a dipole parallel to the surface, the emitted power would increase by more than a factor of 20 .

In reality, the situation is better than suggested by the above calculation, which is correct only for the point dipole formed when the laser spot is small compared to the $\mathrm{THz}$ wavelength $(\approx 300 \mu \mathrm{m}$ in air). For a larger spot, the in-plane coherence across the emitting region tends to collimate the emitted $\mathrm{THz}$ radiation in the same direction as the reflected laser light. ${ }^{18}$ Thus, by increasing the spot size, one can extract a greater fraction of the $\mathrm{THz}$ power. The excited spot can be considered a distribution of point dipoles, with relative phases set by the phase variation across the laser pulse, causing collimation in the reflection direction. Therefore, as the angle of incidence (and hence of the emission) is varied, the power obtained in the collimated direction follows closely the emission pattern for the point dipole, as was previously demonstrated experimentally. ${ }^{19}$ This means that, even with a large 
laser spot, it remains highly beneficial to reorient the dipole relative to the surface.

To reorient the dipole, we placed a prism with a matching refractive index on top of the semiconductor such that the dipole was at an angle of $45^{\circ}$ to the prism surface. However, because of the collimation effect, it was also necessary to ensure that the laser pulse was incident upon the semiconductor at the same angle. We achieved this by using a prism with two $45^{\circ}$ facets, passing the laser through one and collecting the $\mathrm{THz}$ pulse through the other [see Fig. 1(b), inset].

The prism was fabricated by use of molecular beam epitaxy to deposit a 500-nm-thick layer of crystalline InAs onto a $500-\mu \mathrm{m}$ semi-insulating (100) GaAs wafer. The InAs layer was found to be $n$ type, doped with a carrier density of $5 \times 10^{16} \mathrm{~cm}^{-3}$ at room temperature. The wafer was cleaved into $1.5-\mathrm{mm}$ strips, which were glued, epilayer down, onto a brass polishing block. Two facets of the prism were then polished with diamond pastes to form a right-angle prism. Finally, measurements were performed at room temperature, with the device suspended in air, and one of the unpolished strips was used as a (planar) control sample.

Pulses from a Ti:sapphire laser (wavelength, $920 \mathrm{~nm}$; TM polarized; pulse length, $140 \mathrm{fs}$; pulse energy, $2.5 \mathrm{~nJ}$; pulse repetition rate, $86 \mathrm{MHz}$ ) were incident at $45^{\circ}$ onto the back surface of the control and focused to form a Gaussian intensity profile $(\sigma=45 \mu \mathrm{m})$. The laser pulses had a photon energy below the bandgap of GaAs but above that of InAs, so the pulses passed through the GaAs before being absorbed in the InAs. The $\mathrm{THz}$ transient was thus generated in the InAs, but, because the index mismatch was small, there was negligible refraction when it passed into the GaAs.

We obtained the angular power distribution of $\mathrm{THz}$ radiation emitted from the control samples by measuring the voltage of an apertured Golay-cell bolometer (Cathodeon, Cambridge, UK), which was rotated about the sample. The resultant radiation pattern is shown in Fig. 1(a). The two lobes on the polar plot show the weakly collimated $\mathrm{THz}$ beam propagating in the direction of the reflected $\left(45^{\circ}\right)$ and the transmitted $\left(135^{\circ}\right)$ laser beams. Similar radiation patterns were previously reported for bulk surface-field emitters. ${ }^{5}$ The measured power was identical to that obtained from the surface of an InAs bulk wafer under the same excitation conditions.

The prism sample was excited under the same conditions as for the planar control, with the laser at normal incidence to one of the polished facets, giving an internal angle at the InAs interface of $45^{\circ}$. The $\mathrm{THz}$ emission was then collimated about a direction $45^{\circ}$ to the dipole axis and emerged normal to the other prism facet. The measured radiation pattern is shown in Fig. 1(b). The peak THz angular intensity was 8.7 times greater than for a planar reference sample. Furthermore, using $f / 3$ collection optics to collect $\mathrm{THz}$ radiation emitted within a cone of $9.5^{\circ}$ yielded a total power $(\approx 7 \mu \mathrm{W})$ that was 21 times greater for the prism. Indeed, the angularly broad emission from the prism resulted in considerably larger enhancement factors when even smaller $f$-number-optics were used.

Figure 2 shows the electric field of the $\mathrm{THz}$ transient produced by the prism emitter. The shape of the $\mathrm{THz}$ pulse emitted from the prism is similar to the weaker pulse shape measured from the reference sample and from a bulk InAs wafer under the same conditions. These results demonstrate the coherent nature of the generated radiation and show that the prism does not modify the desirable half-cycle waveform of the $\mathrm{THz}$ pulse. The inset of Fig. 2 shows the power spectrum of the emitted pulse.

Other approaches have been used to produce high power pulses. For example, it has been known for several years that the power generated at semiconductor surfaces can be enhanced by approximately an order of magnitude by the application of a large (3-8-T) magnetic field. ${ }^{9-14}$ This effect has been explained (a)
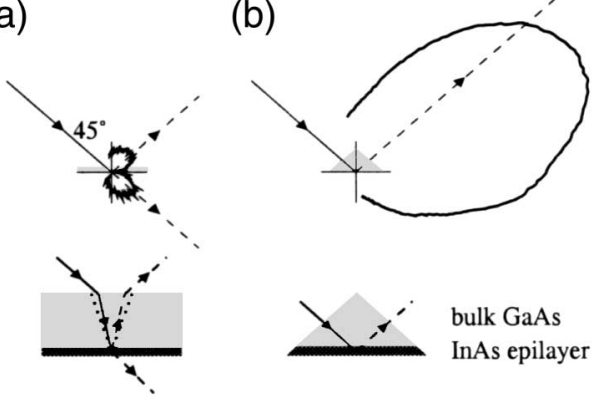

Fig. 1. Polar plots (with the same scaling) showing radiation patterns of emitted $\mathrm{THz}$ power in different sample geometries. The radial distance from the origin is proportional to the $\mathrm{THz}$ power per unit solid angle. The angular resolution of the measurement system was $2^{\circ}$. Below each figure is a schematic diagram of the sample, showing the refracted rays within the structure. The dotted curves (left) show the $16^{\circ}$ light cone in the GaAs layer.

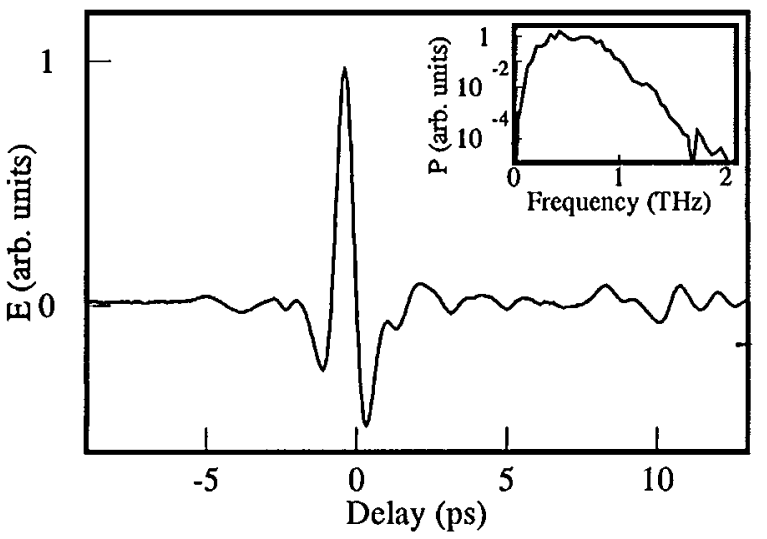

Fig. 2. Time dependent electric field of a $\mathrm{THz}$ transient emitted from the prism sample. The measurement was performed at room temperature by electro-optic sampling in a 1-mm-thick ZnTe crystal. The oscillations after the half-cycle pulse are associated with water vapor absorption. Inset, the power spectrum calculated from these data. 
theoretically as being a consequence of the rotation of the $\mathrm{THz}$ dipole that results from the Lorentz force's acting on the moving carriers. ${ }^{20-22}$ Our ability to produce a similar power enhancement by using the prism geometry provides an experimental confirmation of this explanation. In contrast to the prism device, magnetic-field-enhanced emitters have the disadvantage that producing high magnetic fields requires large magnets, which limits the use of these emitters in many applications.

Other common $\mathrm{THz}$ generation techniques require the application of a voltage across a $\mathrm{p}-\mathrm{i}-\mathrm{n} \operatorname{diode}^{7}$ or between metal electrodes on the surface of a semiconductor (photoconductive switch). ${ }^{23}$ The $\mathrm{p}-\mathrm{i}-\mathrm{n}$ diode emitters have been shown to produce extremely broadband $\mathrm{THz}$ radiation (as much as $40 \mathrm{THz}$ ), ${ }^{7}$ but they suffer from the same dipole orientation problem as the planar sample studied here. Inasmuch as the prism effect is purely geometrical, independently of the microscopic process by which the dipole is formed, it should be possible to obtain gains similar to those reported here. However, the surface electrodes of the photoconductive switch separate the carriers along the surface, so the $\mathrm{THz}$ dipole is formed in the favorable parallel direction, and the gains achievable by improving the output coupling are likely to be much smaller. We expect that, for comparable dipole strengths, the emitted power obtained from prism and photoconductive switch devices will be similar.

To conclude, we have shown that a prism can be used to improve greatly the power of a $\mathrm{THz}$ pulse generated at an optically excited InAs surface. The power levels are comparable to those previously obtained by use of high magnetic fields. The same improvement in $\mathrm{THz}$ generation efficiency should also be obtained in other material systems and with $\mathrm{p}-\mathrm{i}-\mathrm{n}$ diodes. The latter structures are particularly attractive, as they can be designed such that all the photoexcited carriers are generated in the high field region, leading to a larger $\mathrm{THz}$ dipole. The improved output coupling provided by our technique thus provides the opportunity to produce very high-power, low-noise $\mathrm{THz}$ sources that are tunable over a wide bandwidth.

The authors thank Ray Flaxman for technical assistance with polishing and the Rutherford Appleton Laboratory for the loan of a laser. E. H. Linfield and A. G. Davies acknowledge support from Toshiba Research Europe, Ltd., and the Royal Society, respectively. This research was partially funded by the Engineering and Physical Science Research Council (UK). M. B. Johnston's e-mail address is mbj20@cam.ac.uk.

\section{References}

1. C. Waschke, H. G. Roskos, R. Schwedler, K. Leo, H. Kurz, and K. Köhler, Phys. Rev. Lett. 70, 3319 (1993).

2. R. Huber, F. Tauser, A. Brodschelm, M. Bichler, G. Abstreiter, and A. Leitenstorfer, Nature 414, 286 (2001).

3. M. Brucherseifer, M. Nagel, P. H. Bolivar, H. Kurz, A. Bosserhoff, and R. Buttner, Appl. Phys. Lett. 77, 4049 (2000).

4. D. Arnone, C. Ciesla, and M. Pepper, Phys. World 13, 35 (2000).

5. X. C. Zhang and D. H. Auston, J. Appl. Phys. 71, 326 (1992).

6. R. Kersting, K. Unterrainer, G. Strasser, H. F. Kauffmann, and E. Gornik, Phys. Rev. Lett. 79, 3038 (1997).

7. A. Leitenstorfer, S. Hunsche, J. Shah, M. C. Nuss, and W. H. Knox, Appl. Phys. Lett. 74, 1516 (1999).

8. R. Köhler, A. Tredicucci, F. Beltram, H. E. Beere, E. H. Linfield, A. G. Davies, D. A. Ritchie, R. C. Iotti, and F. Rossi, Nature 417 156, 2002 ().

9. N. Sarukura, H. Ohtake, S. Izumida, and Z. Liu, J. Appl. Phys. 84, 654 (1998).

10. S. Izumida, S. Ono, Z. Liu, H. Ohtake, and N. Sarukura, Appl. Phys. Lett. 75, 451 (1999).

11. R. McLaughlin, A. Corchia, M. B. Johnston, Q. Chen, C. M. Ciesla, D. D. Arnone, G. A. C. Jones, E. H. Linfield, A. G. Davies, and M. Pepper, Appl. Phys. Lett. 76, 2038 (2000).

12. C. Weiss, R. Wallenstein, and R. Beigang, Appl. Phys. Lett. 77, 4160 (2000).

13. J. N. Heyman, P. Neocleous, D. Hebert, P. A. Crowell, T. Miller, and K. Unterrainer, Phys. Rev. B 64, 085202 (2001).

14. H. Ohtake, S. Ono, M. Sakai, Z. Liu, T. Tsukamoto, and N. Sarukura, Appl. Phys. Lett. 76, 1398 (2000).

15. T. Dekorsy, T. Pfeifer, W. Kütt, and H. Kurz, Phys. Rev. B 47, 3842 (1993).

16. H. Dember, Physik. Z. 32, 554 (1931).

17. T. Dekorsy, H. Auer, H. J. Bakker, H. G. Roskos, and H. Kurz, Phys. Rev. B 53, 4005 (1996).

18. B. B. Hu, J. T. Darrow, X. C. Zhang, D. H. Auston, and P. R. Smith, Appl. Phys. Lett. 56, 886 (1990).

19. M. B. Johnston, A. Corchia, A. Dowd, E. H. Linfield, A. G. Davies, R. McLaughlin, D. D. Arnone, and M. Pepper, Physica E 13, 896 (2002).

20. J. Shan, C. Weiss, R. Wallenstein, R. Beigang, and T. F. Heinz, Opt. Lett. 26, 849 (2001).

21. M. B. Johnston, D. M. Whittaker, A. Corchia, A. G. Davies, and E. H. Linfield, J. Appl. Phys. 91, 2104 (2002).

22. M. B. Johnston, D. M. Whittaker, A. Corchia, A. G. Davies, and E. H. Linfield, Phys. Rev. B 65, 165301 (2002).

23. D. H. Auston and K. P. Cheung, J. Opt. Soc. Am. B 2, 606 (1984). 